\title{
Three-dimensional analysis of flow and segregation in vertical Bridgman crystal growth under a transversal magnetic field with ampoule rotation
}

\author{
C.W. Lan*, B.C. Yeh \\ Department of Chemical Engineering, National Taiwan University, Taipei 10617, Taiwan, ROC
}

\begin{abstract}
We investigate the flow and solute segregation in a vertical Bridgman crystal growth under a transversal magnetic field with slow ampoule rotation using three-dimensional numerical simulation. The analysis starts from simple ampoule translation and rotation at zero gravity showing the role of magnetic centrifugal pumping caused by the electrically conductive crystal. Then, the analysis is extended to a typical terrestrial growth, and it is found that the rotation improves significantly the axisymmetry of the solute transport at the interface, while the global solute transport is not much affected. The electrical conductivity of both the crystal and the ampoule are found important to the flow and dopant segregation.
\end{abstract}

(C) 2004 Elsevier B.V. All rights reserved.

PACS: 44.25.+f; 47.27.Te; 81.10.Fq; 02.60.c6; 02.70Fj

Keywords: A1. Convection; A1. Lorentz force; A1. Magnetic field; A1. Rotation; A1. Segregation; B2. Bridgman method

\section{Introduction}

The control of heat flow, dopant segregation, and the growth interface by external forces has been found effective in bulk crystal growth [1-9]. The suppression of melt convection to achieve the so-called diffusion-limited growth is particularly important to reduce axial segregation. By further manipulating the residual flow near the interface, radial dopant segregation can be controlled as well. For electrically conductive melt, the magnetic field has been widely used to control the growth [3-9]. Both the static [3-6] and rotating magnetic

\footnotetext{
*Corresponding author. Fax: + 886-2-2363-3917

E-mail address: cwlan@ntu.edu.tw (C.W. Lan).
}

fields (RMF) [7-9] have been reported. For vertical Bridgman growth, the axial field has been widely investigated and used [5]. However, in a recent study by Lan et al. [10], it was shown that the axial field tends to stretch the flow cells axially leading to a better global mixing. Unless the flow can be sufficiently suppressed, the bulk dopant mixing cannot be reduced. Through both steadystate and time-dependent simulations, they also found that the transversal field is more effective in reducing the bulk mixing. Unfortunately, the highly asymmetric dopant mixing near the interface causes severe radial segregation. On the other hand, the RMF is to enhance bulk mixing by combining the transversal magnetic field and rotation. The enhanced convection is often strong 
enough to inverse the growth interface from concave to convex [7]. Owing to its unsteady feature, the rotating rate is usually high being about $50 \mathrm{~Hz}$ or higher. For a low-speed RMF, Walker [11] investigated, based on an asymptotic analysis, the flow structure in a simple Bridgman configuration (isothermal). He concluded that severe segregation could be induced due to the nonuniform flow near the growth interface. For such an RMF configuration, a steady state can exist only when the interface is axisymmetric. Interestingly, from the heat flow point of view, under a stationary transversal magnetic field, rotating the ampoule allows a steady growth, even with an asymmetric heating or growth interface. The dopant field in the crystal is still time dependent, but the angular variation may be reduced by solid-state diffusion at high temperature. However, such an interesting configuration has not yet been investigated so far for the vertical Bridgman (VB) or gradient-freeze growth.

The melt flow structure in a rotating ampoule under a transversal field is believed to be similar to that in RMF if a rotating coordinate frame is adopted [11]. In such a configuration, an electric current passing through the growth interface can be induced, and the Lorentz force can drive a centrifugal pumping flow. Such an angular flow may improve the asymmetric nonuniformity near the interface due to the transversal field. Furthermore, the centrifugal pumping effect is also affected by the crystal conductivity. Although it has not yet been studied by growth experiments, its 3D simulation in a self-consistent manner should be helpful in getting a deeper insight of the flow and segregation control.

In this paper, we present for the first time the effects of slow ampoule rotation in a vertical Bridgman configuration under a transversal magnetic field through 3D simulation analysis. The effects of gravity, as well as the crystal and ampoule electrical conductivities, on the flow structures and dopant segregation are illustrated. In the next section, the numerical model is briefly described. Section 3 is devoted to results and discussion, followed by conclusions in Section 4.

\section{Mathematical model and numerical solution}

The mathematic model and the numerical solution used here are the same as the one used by Lan et al. [10]. In short, a Cartesian coordinate $(x, y, z)$ with the origin (fixed and stationary) at the sample bottom is chosen, as shown in Fig. 1 ; the $x$ axis is at the centerline of the sample. The furnace thermal profile is assumed to be linear, and the effect of thermal radiation is included in the effective heat transfer coefficient [12]. The ampoule is moving downward at a speed $U_{\text {amp }}$ and is rotating at a constant speed $\Omega$. To present the variables in dimensionless form, the length is scaled by ampoule length $L$, time by $L^{2} / \alpha_{\mathrm{m}}$, velocity by $\alpha_{\mathrm{m}} / L$, concentration by $C_{0}$, temperature by the temperature difference $\Delta T\left(\Delta T=T_{\mathrm{H}}-\right.$ $T_{\mathrm{C}}$ ), and electrical potential by $\alpha_{\mathrm{m}} B_{0}$, where $\alpha_{\mathrm{m}}$ is the thermal diffusivity, $C_{0}$ the artificial inlet concentration at the top surface (for pseudosteady-state calculations) [10], and $B_{0}$ the applied magnetic field strength. $T_{\mathrm{H}}$ and $T_{\mathrm{C}}$ are the hotand cold-zone temperatures, respectively, as shown in Fig. 1. Then, with the Boussinesq approximation, the dimensionless governing equations for the transport processes in the melt during crystal growth can be described by the conserva-

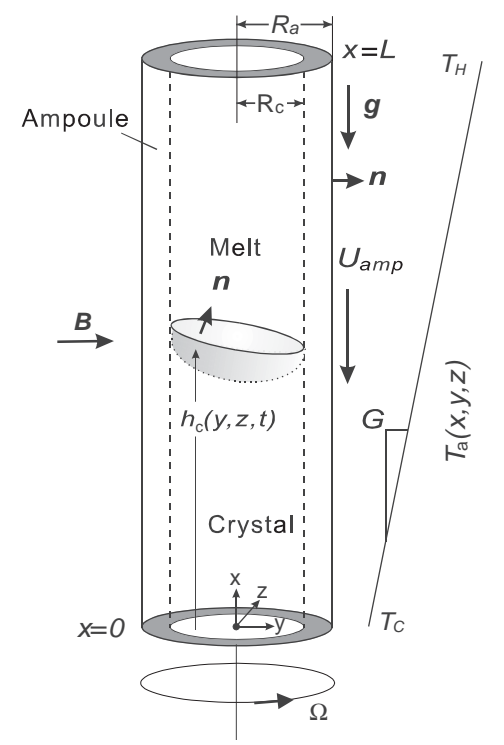

Fig. 1. Schematic of a vertical Bridgman crystal growth under a transversal magnetic field with ampoule rotation. 
tion laws for the mass, momentum, energy, dopant, and the electrical current density as follows [10]:

$\boldsymbol{\nabla} \cdot \boldsymbol{v}=0$,

$$
\begin{aligned}
\frac{\partial \boldsymbol{v}}{\partial \tau}+\boldsymbol{v} \cdot \nabla \boldsymbol{v}= & -\nabla P+\operatorname{Pr} \nabla^{2} \boldsymbol{v}+\operatorname{Pr} R a_{\mathrm{T}}\left(\theta-\theta_{\mathrm{m}}\right) \boldsymbol{e}_{x} \\
& +\operatorname{PrHa}^{2}\left(-\nabla \Phi+\boldsymbol{v} \times \boldsymbol{e}_{\mathrm{B}}\right) \times \boldsymbol{e}_{\mathrm{B}},
\end{aligned}
$$

$\frac{\partial \theta}{\partial \tau}+\boldsymbol{v} \cdot \nabla \theta=\nabla^{2} \theta$

$\frac{\partial C}{\partial \tau}+\boldsymbol{v} \cdot \nabla C=\frac{P r}{S c} \nabla^{2} C$

$\nabla \cdot \sigma_{i}^{*} \nabla \Phi=\nabla \cdot \sigma_{i}^{*}\left(\boldsymbol{v} \times \boldsymbol{e}_{\mathrm{B}}\right)$,

where, $v, \tau, P, \theta, C, \Phi$ are the dimensionless velocity, time, pressure, temperature, dopant concentration, and electric field, respectively; $\theta_{\mathrm{m}}$ is the dimensionless melting temperature. $P r=v_{\mathrm{m}} / \alpha_{\mathrm{m}}$ is the Prandtl number and $S c=v_{\mathrm{m}} / D$ the Schmidt number, where $v_{\mathrm{m}}$ is the kinematic viscosity and $D$ the dopant diffusivities. Also, $\boldsymbol{e}_{x}$ is the unit vector in the axial direction and $\boldsymbol{e}_{\mathrm{B}}$ the magnetic field direction, which is set to be transversal here, i.e., $\boldsymbol{e}_{\mathrm{B}}=\boldsymbol{e}_{y}$, where $\boldsymbol{e}_{y}$ is the unit vector in the $y$ direction. Furthermore, $\sigma_{i}^{*}=\sigma_{i} / \sigma_{\mathrm{m}}, i=(\mathrm{m}, \mathrm{c}$, amp) is the normalized electrical conductivity of the melt (m), the crystal (c), and the ampoule (amp). In the body force term of Eq. (2), the centrifugal acceleration [13] is not considered because of low rotation rate used here. Eqs. (3) and (5) are also applied to the ampoule and the crystal with different physical properties.

In addition, the associated dimensionless thermal Rayleigh $\left(R a_{\mathrm{T}}\right)$ and Hartmann $(H a)$ numbers are defined as the following:

$R a_{\mathrm{T}}=\frac{\beta_{\mathrm{T}} g_{0} \Delta T L^{3}}{v_{\mathrm{m}} \alpha_{\mathrm{m}}} ; H a=B_{0} L\left(\sigma_{\mathrm{m}} / \mu_{\mathrm{m}}\right)^{1 / 2}$,

where $\beta_{\mathrm{T}}$ is the thermal expansion, $g_{0}$ the gravitational acceleration, and $\mu_{\mathrm{m}}$ the melt viscosity. Also, the magnetohydrodynamic (MHD) equations used above are the same as the MHD2 model used by Baumgartl and Müller [14].

In this report, to focus on the interplay of the flow, ampoule rotation, and the magnetic field, pseudo-steady-state calculations are presented only. The physics for time-dependent growth should be similar [10]. To solve the above equations, a set of proper boundary conditions is required. In short, zero normal electrical current at the outer ampoule wall and the current continuity at the interfaces are imposed for the calculation of the electrical potential. For velocity, the no-slip boundary condition is adopted at the melt/solid interface, i.e., $\boldsymbol{v}=v_{\mathrm{amp}} \boldsymbol{e}_{x}+0.5 \mathrm{Ta}^{1 / 2} \operatorname{Prre}_{\theta}$, where $v_{\mathrm{amp}}$ is the dimensionless pulling speed by assuming the same melt and crystal density, the Taylor number $T a=4 \Omega^{2} L^{4} / v_{\mathrm{m}}^{2}$, and $\boldsymbol{e}_{\theta}$ the unit vector in the azimuthal angle direction. Other boundary conditions for pressure, temperature and dopant concentration, as well as the conditions at the interfaces, can be found elsewhere [10,12,13]. Finally, the above governing equations are solved numerically by an efficient finite volume method with multigrid acceleration [15].

\section{Results and discussion}

For comparison purposes, we take the growth of gallium-doped germanium $(\mathrm{GaGe})$ in a graphite ampoule as an example. This problem, without magnetic fields, was also investigated by Adornato and Brown [16]. Extensive benchmark numerical comparisons were also performed [12,17]. Therefore, it serves as a good candidate for the theoretical study here. The situation of ampoule rotation in an axial magnetic field has also been studied based on an axisymmeytric assumption [18]. The physical properties and some input parameters $\left(R_{\mathrm{c}}=0.5 \mathrm{~cm}, R_{\mathrm{a}}=0.7 \mathrm{~cm}, L=7 \mathrm{~cm}\right.$, and $\Delta T=350^{\circ} \mathrm{C}$ ) can be found elsewhere [16-18]; $\sigma_{m}=1.66 \times 10^{4} / \Omega / \mathrm{cm}, \sigma_{\mathrm{c}}=1.11 \times 10^{3} / \Omega / \mathrm{cm}$, and $\sigma_{\mathrm{amp}}=2.533 \times 10^{4} / \Omega / \mathrm{cm}$. The corresponding dimensionless numbers are: $S c=6.19, P r=0.00715$, $R a_{\mathrm{T}}=2.491 \times 10^{8}, \quad$ and $H a=1685.43$ for $g_{0}=981 \mathrm{~cm} / \mathrm{s}^{2}$ and $B_{0}=0.5 T$. In fact, this problem is quite ideal for most of gradient-free (or VB) growth that the interface is concave due to the smaller crystal thermal conductivity than the melt. The melt convection (toroidal-cell flow) is generated above the interface due to radial thermal gradients, while the convection in the bulk melt remains weak. Under a transversal field, the 
problem is fully $3 \mathrm{D}$, and no axisymmetric solution is available.

Before showing a more realistic growth condition, the basic flow and solute fields for a translating and rotating system at zero gravity in a transversal field is examined first. Fig. 2 shows the effects of 10 RPM ampoule rotation on the flow and solute fields under a transversal field of $0.5 \mathrm{~T}$. Without ampoule rotation in Fig. 2a, the velocity vectors are simply due to ampoule translation, and the solute distribution is purely diffusive, which is counterbalanced by the downward melt flow. The radial solute segregation is caused by the interface concavity, but the distribution is still axisymmetric. With ampoule rotation of 10 RPM, as shown in Fig. 2b, convection cells are induced. Although the flow is quite weak, the radial segregation profiles are affected. Interestingly, the radial segregation is reduced with the increasing rotation speed. Such flow cells are due to the centrifugal magnetic flow pumping, which can be seen clearly if we take the flow on the plane slightly above the interface at $x=3.47 \mathrm{~cm}$. As shown on the top figure of Fig. 2c, there are three flow cells that can be viewed from the top. The center one is in the opposite direction to the ampoule rotation. This centrifugal pumping cell can be realized easily. By taking the $x-y$ plane at $z=0$ as an example, if the crystal is electrically conductive (here $\sigma_{\mathrm{c}}=0.066 \sigma_{\mathrm{m}}$ ), there is a downward electrical current $\left(\boldsymbol{v} \times \boldsymbol{B}=r \Omega \boldsymbol{e}_{z} \times B_{0} \boldsymbol{e}_{y}=\right.$ $-r \Omega B_{0} \boldsymbol{e}_{x}$ ) flowing across the interface at the positive $y$ side (on the right), while an upward current in the other side (on the left), where $\boldsymbol{j}=$ $\boldsymbol{v} \times \boldsymbol{B}=r \Omega \boldsymbol{e}_{z} \times B_{0} \boldsymbol{e}_{y}=r \Omega B_{0} \boldsymbol{e}_{x} ; \quad r$ is the radial distance. Then, the Lorentz force $\boldsymbol{j} \times \boldsymbol{B}=$ $-r \Omega B_{0}^{2} \boldsymbol{e}_{z}$ is generated on the right, while $r \Omega B_{0}^{2} \boldsymbol{e}_{z}$ on the left. They are both opposite to the ampoule rotation and induce a clockwise flow. However, on the $x-z$ plane at $y=0$, because the rotation direction is parallel to the magnetic field, no electrical current or Lorentz force is induced. Therefore, for the same radial distance, the centrifugal pumping force is the highest on the $x-y$ plane and gradually reduces to zero at the $x-z$ plane. Accordingly, in Fig. 2c two cells remain the same direction as the ampoule rotation near the ampoule wall at the top and the bottom due to the weaker centrifugal pumping force there. Such a nonuniform Lorentz force also causes the asymmetric flow, and thus the asymmetric solute distribution, as shown in Fig. 2b. If the electrical
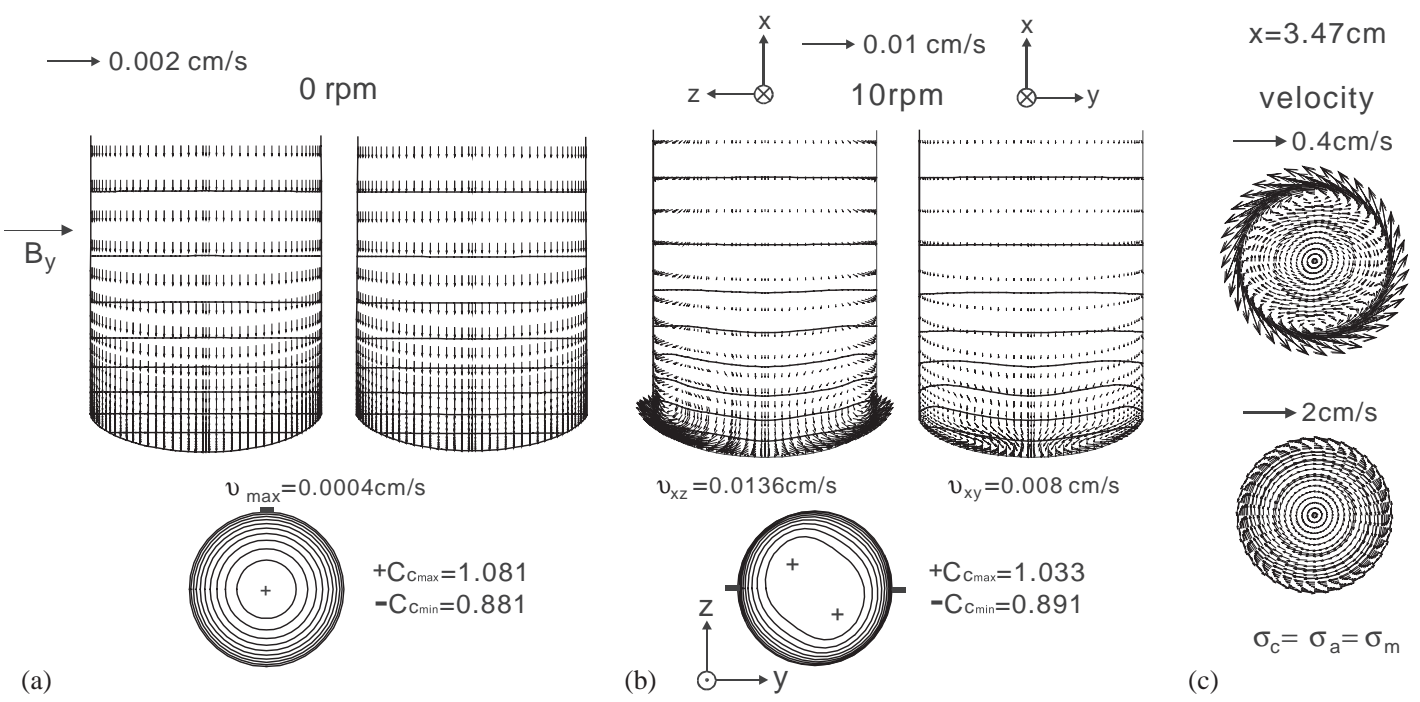

Fig. 2. Effects of ampoule rotation on flow and dopant fields at zero gravity under a transversal field of 0.5 T: (a) 0 RPM; (b) 10 RPM; (c) flow fields at $x=3.47 \mathrm{~cm}$ from (b) (top plot); the bottom plot is the flow field at the same plane but with $\sigma_{\mathrm{c}}=\sigma_{\mathrm{a}}=\sigma_{\mathrm{m}}$. $U_{\mathrm{amp}}=-4 \times 10^{-4} \mathrm{~cm} / \mathrm{s}$ 
conductivity of the crystal is increased to be the same as the melt $\left(\sigma_{\mathrm{c}}=\sigma_{\mathrm{amp}}=\sigma_{\mathrm{m}}=1.66 \times 10^{4} / \Omega /\right.$ $\mathrm{cm}$ ), as shown in the bottom figure of Fig. 2c, the centrifugal pumping flow becomes much stronger and the overall flow is in the clockwise direction beside the thin boundary layer near the ampoule wall; notice that the velocity vector scale is increased here. Such a centrifugal pumping slows down significantly the rotational flow field due to the no-slip boundary condition at the interface and the wall. As a result, the local flow behavior mimics a stationary fluid subjected to crucible rotation causing a downward flow viewed from the side as that shown in Fig. $2 b$.

At normal gravity, the effects of ampoule rotation are slightly different from that at zero gravity, as shown in Fig. 3, due to the existence of thermal convection. Without ampoule rotation, the thermal convection in the $x-y$ plane is significantly suppressed. However, in the $x-z$ plane, due to the buildup of electrical potential, the Lorentz force is much weaker. As a result, the convection is not suppressed much [10]. Such a nonuniform mixing results in a highly asymmetric dopant distribution in the melt and the growing crystal, as illustrated in Fig. 3a. However, with 10 RPM ampoule rotation, as shown in Fig. 3b, the flow fields are greatly modified, and the radial segregation is improved. Much more axisymmetric flow and solute fields are obtained. The top-view of the flow at $x=3.47 \mathrm{~cm}$ is shown on the top figure of Fig. 3c. The same as that in zero gravity, the flow has a three-cell structure. Again, if the electrical conductivity of the crystal is increased to be the same as that in the melt, as shown in the bottom plot of Fig. 3c, a much stronger centrifugal pumping is induced leading to a single cell flow (counterclockwise in direction), whose direction is opposite to the ampoule rotation.

If we summarize the calculations for different magnetic field strengths and rotation speeds at normal gravity, as shown in Fig. 4, we can get a bigger picture on the improvement of the radial (the maximum concentration difference at the interface) and the axial segregations. The axial segregation can be judged through the effective segregation coefficient $K_{\text {eff }}$, which is evaluated by $C_{0} /\langle C\rangle$, where $\langle C\rangle$ is the averaged solute concentration in the melt. For diffusive growth,

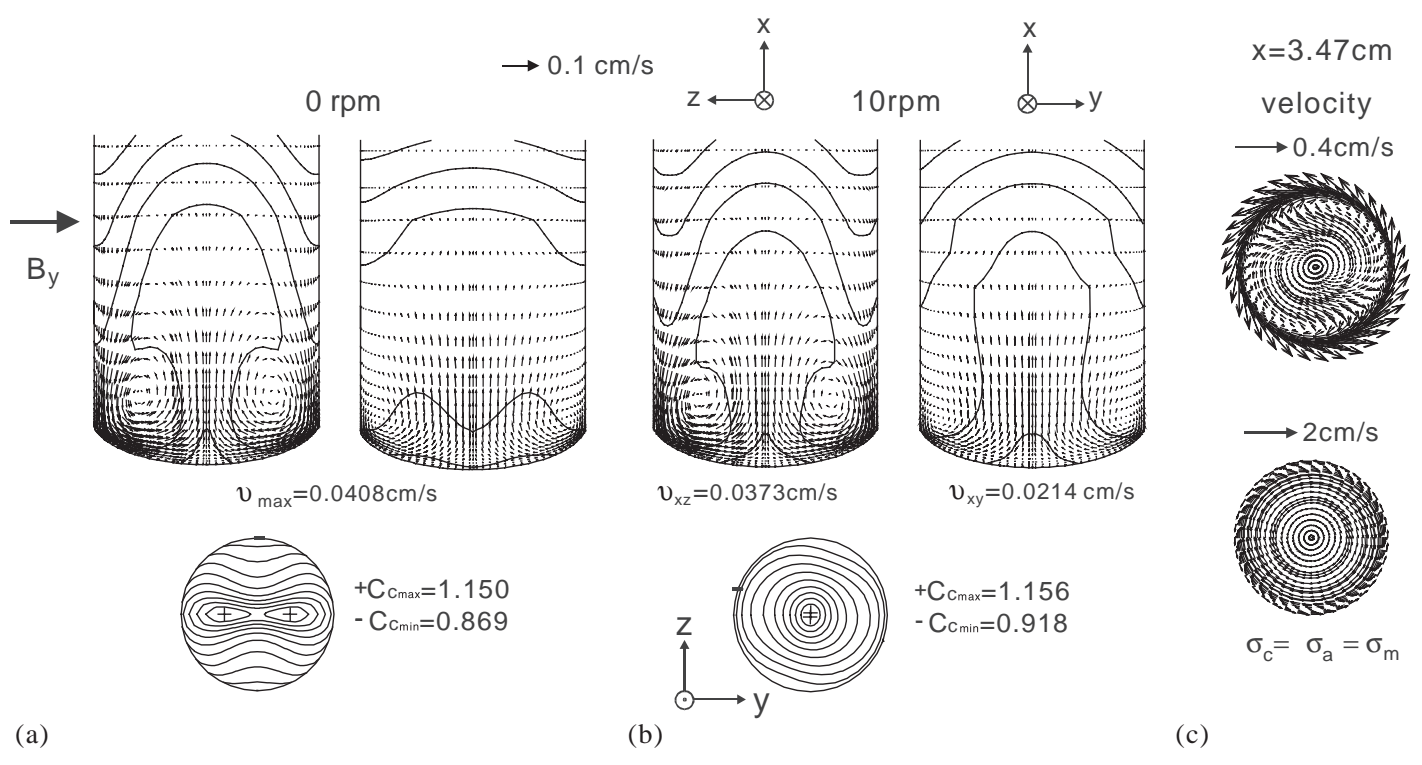

Fig. 3. Effects of ampoule rotation on flow and dopant fields at normal gravity under a transversal field of $0.5 \mathrm{~T}$ : (a) 0 RPM; (b) 10 RPM; (c) flow fields at $x=3.47 \mathrm{~cm}$ from (b) (top plot); the bottom plot is the flow field at the same plane but with $\sigma_{\mathrm{c}}=\sigma_{\mathrm{a}}=\sigma_{\mathrm{m}}$. $U_{\mathrm{amp}}=-4 \times 10^{-4} \mathrm{~cm} / \mathrm{s}$ 

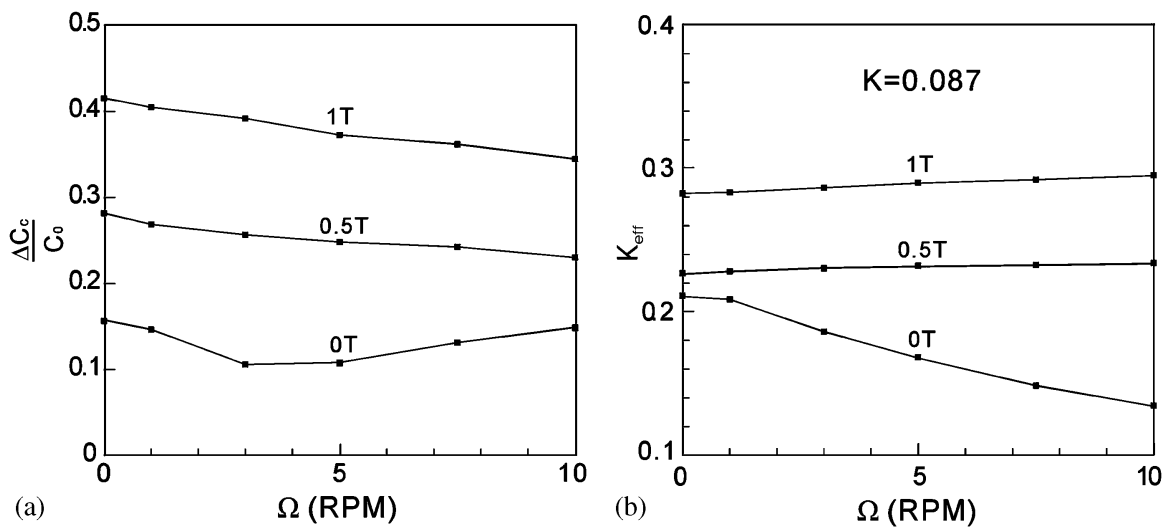

Fig. 4. Effects of rotation on: (a) radial segregation; (b) effective segregation coefficient $K_{\text {eff }} ; U_{\text {amp }}=-4 \times 10^{-4} \mathrm{~cm} / \mathrm{s}$.

i.e., without thermal convection, $K_{\mathrm{eff}}=0.394$. For the case of no magnetic field, as shown in Fig. 4a, the radial segregation reaches a minimum at about 3 RPM. With the magnetic field strength of 0.5 and $1 \mathrm{~T}$, the radial segregation reduces with the rotation speed monotonically. Nevertheless, radial segregation increases when the flow intensity is reduced. Apparently, this is a trade off by the reduction of axial segregation due to magnetic flow suppression. For the effective segregation coefficient, as shown in Fig. 4b, the behavior is interesting. Without the magnetic field, ampoule rotation stretches the flow cell axially leading to a better bulk mixing as discussed before [18]. As a result, the effective segregation decreases with the rotation speed. Of course, there will be a reduction of the global mixing at a much higher rotation speed [13], but it is beyond the scope of present discussion. Interestingly, with the magnetic field of 0.5 and $1 \mathrm{~T}$, the effective segregation coefficient increases very slowly with the ampoule rotation. This is a clear evidence that the rotation does not enhance the global dopant mixing. On the contrary, it reduces slightly the axial segregation. For the same magnetic field strength, the radial segregation is reduced as well by rotation (Fig. 4a). Therefore, it may be concluded that under a transversal field, a proper ampoule rotation could be useful in getting a better control of the flow and dopant segregation.

\section{Conclusions}

In this study, we have performed 3D calculations to investigate the influence of slow ampoule rotation under a transversal field on the flow and dopant fields in a vertical Bridgman crystal growth. It is found that with ampoule rotation the centrifugal magnetic melt pumping could be significant and could generate convection even at zero gravity. Such a flow seems to be beneficial in reducing radial segregation under the same magnetic field strength. At normal gravity, the transversal field, though suppresses the flow, produces nonuniform flow and dopant fields. However, with ampoule rotation, the nonuniformity could be greatly improved. As a result, both radial and axial segregation could be reduced. Furthermore, the centrifugal melt pumping increases with the increasing crystal conductivity, and further enhances the dopant mixing (in the angular direction) near the growth interface, which is useful in improving homogeneity. Nevertheless, it should be pointed out that the radial segregation with flow suppression is still larger than that without the magnetic field in this study. At this medium magnetic field strength $(0.5-1 \mathrm{~T})$, the advantage of the flow suppression is only on the smaller axial segregation due to the larger effective segregation coefficient. To reduce both radial and axial segregation, a stronger magnetic field seems to be inevitable. 


\section{References}

[1] R.A. Brown, AIChE J. 34 (1989) 881.

[2] G. Müller, A. Ostrogorsky, in: D.T.J. Hurle (Ed.), Handbook of Crystal Growth 2b: Growth Mechanisms and Dynamics, North-Holland, Amsterdam, 1994.

[3] H.P. Utech, M.C. Flemming, J. Appl. Phys. 37 (1966) 2021.

[4] K.M. Kim, J. Electrochem. Soc. 132 (1982) 427.

[5] D.H. Kim, P.M. Adornato, R.A. Brown, J. Crystal Growth 89 (1988) 339.

[6] D.T.J. Hurle, G. Müller, R. Nitsche, in: H.U. Walter (Ed.), Fluid Sciences and Materials Science in Space, A European Perspective, Springer, Berlin, 1987, p. 315.

[7] Yu.M. Gelfgat, J. Crystal Growth 198/199 (1999) 165.

[8] P. Dold, K.W. Benz, Progress in Crystal Growth and Characterization of Materials, 1997, pp. 7-38.
[9] O. Patzold, I. Grants, U. Wunderwald, K. Jenkner, A. Croll, G. Gerbeth, J. Crystal Growth 245 (2002) 237.

[10] C.W. Lan, I.F. Lee, B.C. Yeh, J. Crystal Growth 254 (2003) 503.

[11] J.S. Walker, J. Crystal Growth 192 (1998) 318.

[12] M.C. Liang, C.W. Lan, J. Crystal Growth 167 (1996) 320.

[13] C.W. Lan, C.Y. Tu, J. Crystal Growth 226 (2001) 406.

[14] J. Baumgartl, G. Müller, Proceedings of the 8th Symposium on Materials and Fluid Science in Microgravity, ESA Publication No. SP333, Brussels, 1992, p.161.

[15] C.W. Lan, M.C. Liang, J. Comp. Phys. 152 (1999) 55.

[16] P.M. Adornato, R.A. Brown, J. Crystal Growth 152 (1987) 155.

[17] C.W. Lan, F.C. Chen, Comput. Methods Appl. Mech. Eng. 131 (1996) 191.

[18] C.W. Lan, J. Chin. Inst. Chem. Engrs. 31 (2000) 465. 